Journal of Animal and Veterinary Advances 11 (11): 1837-1842, 2012

ISSN: $1680-5593$

(C) Medwell Journals, 2012

\title{
Expression of Deiodinase Gene $m R N A$ after Melatonin Manipulated in Cashmere Goats Skin During Cashmere Growth
}

\author{
${ }^{1,2}$ Chunwang Yue, ${ }^{1}$ Maohong Sun, ${ }^{3}$ Haiying Liu, ${ }^{2}$ Wei Zhang, \\ ${ }^{2}$ Xiaoping Zhu, ${ }^{1}$ Xianghao Kong and ${ }^{2}$ Zhihai Jia \\ ${ }^{1}$ College of Animal Science and Technology, Hebei North University, 075000 Zhangiiakou, China \\ ${ }^{2}$ College of Animal Science and Technology, China Agricultural University, 100094 Beijing, China \\ ${ }^{3}$ College of Animal Science and Veterinary Science, Shenyang Agricultural University, \\ 110866 Shenyang, China
}

\begin{abstract}
The aim of this research was to investigate the changes of the Monodeiodinase (MD) gene $m R N A$ in skin of cashmere goats from the initiation of cashmere fibre growth to normal growth. The Melatonin (MT) implants were used in order to initiate growth of cashmere fibre before normal time. Real-time reverse transcription quantitative Polymerase Chain Reaction (real-time PCR) was used to determine the MD gene $m R N A$ expression levels of skin. The results show that the MT concentration was significant differences after administration from July to November the cashmere fibre growth rate was markedly greater in group of MT implant ( $\mathrm{M}$ group) than that of the group of control (C group) in July ( $\mathrm{p}<0.01$ ), August ( $<<0.001$ ) and September $(\mathrm{p}<0.05$ ), the expression of cashmere goat skin MDII mRNA is gradually increased from June to September and then decreased gradually after the implant MT MDII mRNA expression increased rapidly, $\mathrm{M}$ and $\mathrm{C}$ group in July was significantly different $(p<0.01)$ and August are still significant differences $(p<0.05)$, the $M$ and $C$ group MDIII mRNA expression showed a downward trend from June to November in Cashmere goat skin and the M and $\mathrm{C}$ group did not appear significant differences $(\mathrm{p}>0.05)$ in the monthly. These results suggest that the expression levels of MDII mRNA may be involved in the process of cashmere growth, it expression may have a specific signaling pathway in cashmere goats skin, especially in the initiation of cashmere fibre growth period.
\end{abstract}

Key words: Monodeiodinase, gene $m R N A$ expression, real-time RT-PCR, melatonin, cashmere fibre, cashmere goats

\section{INTRODUCTION}

In cashmere goats, the pattern of growth and moult of the cashmere is seasonal with growth generally beginning after the longest day (the Summer solstice) and moult occurring after the shortest (the Winter solstice) (Santiago-Moreno et al., 2004). Studies have already indicated that marked changes of the concentration several hormones that are related to hair follicle activity, cashmere fibre growth and moult (Kloren et al., 1993; Dicks et al., 1994; Santiago-Moreno et al., 2005). Previous studies have shown that lack of thyroid hormone in the hair follicle to reduce the rate of cell division resulting in decline in wool growth rate and yield reduction (Hynd et al., 1994). However, Kloren studies have shown that thyroid hormone on the performance of cashmere goats did not affect (Kloren and Norton, 1995) but later research showed cashmere growth rate and plasma thyroid hormone (T3, T4) were positively correlated
(Rhind and McMillen, 1995). These findings appear inconsistent because it is complex due to hormonal regulation mechanisms.

The expression of Monodeiodinase (MD) has been investigated; the Monodeiodinase type II (MDII) and the Monodeiodinase type III (MDIII) were shown to be present in the skin of cashmere goats. However, the Monodeiodinase type I (MDI) was not detected (Villar et al., 2000). In Scottish cashmere goats, Rhind et al. (2004) found seasonal variation in MD expression with a lower level of activity of MDII during periods of long daylength (July and April) and a higher level in December. Researchers have research to prove that the skin $\mathrm{MD}$ activity showed a seasonal variation in Chinese Inner Mongolia cashmere goats with the MDII activity increasing and the MDIII and MDIII/MDII ratio decreasing from June (the Summer solstice) to November (Yue et al., 2007). Researchers speculated that differences between individual and genotype in hair follicle activity

Corresponding Author: Zhihai Jia, College of Animal Science and Technology, China Agricultural University, 100094 Beijing, China 
and cashmere fibre growth were partially dependent on the pattern of expression of $\mathrm{MD}$ in the skin. Therefore, researchers need further research in this area, at the molecular level to explore the relationship between $\mathrm{MD}$ and hair growth. Therefore, we will investigate skin tissue gene expression in the $\mathrm{MD}$ from the initiation of cashmere fibre growth to normal growth.

In addition, exogenous MT can initiate growth of cashmere fibre and also can stimulate the fibre elongation of secondary follicles in vitro (Ibraheem et al., 1994) and histological examination of skin also showed that fibre growth was initiated by MT treatment (Nixon et al., 1993; Dicks et al., 1996). The results of research also demonstrate that MT played an important role in the regulation of skin $\mathrm{MD}$ activity (Yue et al., 2007). So, in this research, we are still in cashmere goats of subcutaneous implants of MT to stimulate germination of cashmere fiber in advance.

The aim of this research was to investigate the changes of the level of MDII mRNA and MDIII mRNA in skin of cashmere goats from the initiation of cashmere fibre growth to normal growth after MT treatment. For more detailed study T3, T4 and DM role in the growth process of cashmere to provide a basis.

\section{MATERIALS AND METHODS}

Animals and treatment: The study was performed at the stock farm of Inner Mongolia in China (latitude $39^{\circ} 06^{\prime} \mathrm{N}$, longitude $107^{\circ} 59^{\prime} \mathrm{E}$ and at altitude of $1500 \mathrm{~m}$ ). Sixteen halfsib wethers (that had a mean $( \pm \mathrm{SD})$ age of $15 \pm 1$ months and an initial mean $( \pm \mathrm{SD})$ body weight of $33.1 \pm 2.9 \mathrm{~kg}$ ) were allocated randomly to two groups ( $\mathrm{n}=8 /$ group). One group (the $\mathrm{M}$ group) was subcutaneously implanted with the MT implants (2 $\mathrm{mg} \mathrm{kg}^{-1} \mathrm{BW}$; Northeast Forest University, China) at the base of the ear at three 2 monthly intervals commencing in June (the Summer solstice). The other group ( $\mathrm{C}$ group) served as the control. During the experiment period, all goats were grazed on natural pasture. The composition of vegetation in this area has been described (Zhou et al., 2003).

Sample collections, recording and measurement: Blood samples were collected at 10:00 am from all experimental goats by jugular venepuncture at 1 monthly intervals throughout the study. The blood (EDTA anticoagulant) was immediately centrifuged and the plasma frozen at $-20^{\circ} \mathrm{C}$ until assayed. The length of cashmere fibre was recorded monthly. Cashmere fibre growth rate were calculated according to the method of Rhind and McMillen (1995). Skin samples (approximately $1 \mathrm{~cm}^{2}$ ) were cut monthly from the mid-side region under local anaesthesia (Procaine hydrochloride, Huabei Medicine Company, China), snap frozen in liquid nitrogen and then stored at $-70^{\circ} \mathrm{C}$ for subsequent extraction of total RNA.

Hormone assays: Plasma MT concentration was determined using the MT ELISA kit (RE 54021, ImmunoBiological Laboratories GmbH of Hamburg, Germany). The assay procedure was done according to the instructions for use. Intra-assay coefficient of variation and interassay coefficient of variation were 7.2 and $12.9 \%$, respectively.

Extraction of total RNA and reverse transcription: Each sample of skin was first disrupted using a freezer mill (6750-230 Freezer/mill, Spex Co., NJ, USA) and total RNA was extracted from the fine powder using RNAultra kit (Tiangen Biotech Co., Beijing, China). The extract procedure was done according to instructions. RNA was purified from the total RNA using Purification kit (TaKaRa Biotechnology Co., Ltd. Dalian China) according to instructions. RNA concentration was measured by Spectrophotometry at $260 \mathrm{~nm}$ and RNA integrity was verified by agarose gel electrophoresis.

Reverse transcription was carried out in a $50 \mu \mathrm{L}$ final volume containing the total RNA (approximately $3 \mu \mathrm{g}$ ) solution, $200 \mathrm{U}$ M-MLV reverse transcriptase (Promega Co., Madison, Wisconsin, USA), $25 \mathrm{U}$ recombinant ribonuclease inhibitor ( $\mathrm{RNasin}$ ) (TaKaRa Biotechnology Co., Ltd. Dalian China), 5 ng oligo (dT) 18 primer (Promega Co., Madison, Wisconsin, USA) and $2 \mu \mathrm{L}$ dNTP mix $(10 \mathrm{mM})$ according to the manufacturer's instructions. The transcribed cDNA was inspected by PCR using paired primers of housekeeping gene (Glycerolaldehyd-3Phosphate-Dehydrogenase gene, GAPDH). Reaction cycles consisted of an initial denaturing step at $95^{\circ} \mathrm{C}$ for $3 \mathrm{~min}$ followed by 33 cycles of denaturing at $95^{\circ} \mathrm{C}$ for $15 \mathrm{sec}$, annealing at $62.7^{\circ} \mathrm{C}$ for $30 \mathrm{sec}$ and extension at $72^{\circ} \mathrm{C}$ for $30 \mathrm{sec}$ followed by extension at $72^{\circ} \mathrm{C}$ for $7 \mathrm{~min}$ and $4^{\circ} \mathrm{C}$ for ever. The PCR products were inspected by gel electrophoresis.

Oligonucleotide primers were designed using Primer Premier 5.00 Software (Premier Co., Santa Cruz, CA) for MDII, MDIII and GAPDH. The primers were synthesised by professional company (Sangon Biotech Co., Shanghai, China).

Real-time quantitative PCR: Fluorescent real-time quantitative PCR was used to determine mRNA expression levels of skin for total MDII and MDIII. Primer concentrations were optimized and absence of primerdimers was verified before actual mRNA quantification. $\mathrm{PCR}$ reactions for each gene were conducted in a final 
Table 1: Real-time PCR primer sequences and PCR product size for GAPDH, MDII and MDIII

\begin{tabular}{|c|c|c|c|}
\hline Gene & Primers $\left(5^{\prime} \rightarrow 3^{\prime}\right)$ & $\begin{array}{l}\text { Product } \\
\text { (bp) }\end{array}$ & Genbank \\
\hline \multicolumn{4}{|c|}{ GAPDH Forward: GCAAGTTCCACGGCACAG } \\
\hline & -TTC $C A C C O C A$ & 249 & $\mathrm{~J} 4312$ \\
\hline \multirow[t]{2}{*}{$M D I I$} & Forwar & & \\
\hline & & 131 & AB2014 \\
\hline \multirow[t]{2}{*}{$M D I I I$} & & & \\
\hline & GAAGAAATCCA & 144 & \\
\hline
\end{tabular}

volume of $20 \mu \mathrm{L}$ that contained $10 \mu \mathrm{L}$ of SYBR Green PCR Master Mix (Applied Biosystems, Foster City, CA, USA), optimal primer concentrations $(0.4 \mu \mathrm{L}$ each, $200 \mathrm{nM})$ and $2 \mu \mathrm{L}$ cDNA using a Prism 7000 Real Time PCR System (ABI Co., Foster City, California, USA). Real-Time PCR procedure was performed according to the manufacturer's instructions. The real-time PCR primer sequences are shown in Table 1. Relative efficiencies of amplification of MDII, MDIII and GAPDH were validated as approximately equal by running a serial dilution of highly concentration cDNA. Calibrated and non-template controls were include in each assay. Each sample was run in duplicate. SYBR Green dye intensity was analysed using the ABI prism 7000 SDS Software (ABI Co., Foster city, California, USA). All results were normalized to the GAPDH (an unaffected housekeeping gene).

Statistical analysis: Data were analyzed using the t-test and ANOVA procedure of SAS. Significant means were separated using Duncan's multiple range tests.

\section{RESULTS AND DISCUSSION}

Hormone concentrations: Plasma MT concentration showed no significant difference between both groups before administration of MT in June ( $>>0.05)$ but there were significant differences after administration from July to November, the results shown in Fig. 1.

Cashmere fibre growth rate: The $\mathrm{M}$ group cashmere fibre growth rate was markedly greater than that of the $\mathrm{C}$ group in July $(p<0.01)$, August $(p<0.001)$ and September $(\mathrm{p}<0.05)$. There was no significant difference between the $\mathrm{M}$ and $\mathrm{C}$ groups in October, November and December $(\mathrm{p}>0.05)$ (Fig. 2). There was a trend towards increasing cashmere fibre growth rate from July to October in the C group $(\mathrm{p}<0.05)$.

Expression levels of MD mRNA: The result of total RNA integrity verified by agarose gel electrophoresis was shown in Fig. 3. All RNA samples had A260/A280 ratios between 1.7 and 1.9 indicating pure and clean RNA isolates. Primer specificity was analyzed by a comparison of PCR products (Fig. 4) using agarose gel

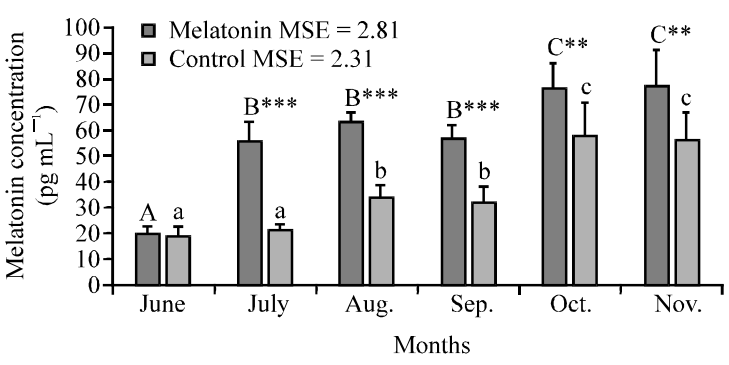

Fig. 1: Both of MT implants groups (M groups) and control groups (C groups) plasma MT concentration ( $\mathrm{pg} \mathrm{mL}^{-1}$ ) at monthly intervals (The samples of June was sampling before implants of MT) from June to November. With different capital letters are significant differences $(\mathrm{p}<0.05)$ in the $M$ groups with different small letter are significant differences $(\mathrm{p}<0.05)$ in the $\mathrm{C}$ groups. The $\mathrm{M}$ groups superscribed with $*$ are significant difference $(p<0.05)$ to the $C$ groups $\left({ }^{* *} \mathrm{p}<0.01\right.$; $* * * \mathrm{p}<0.001)$

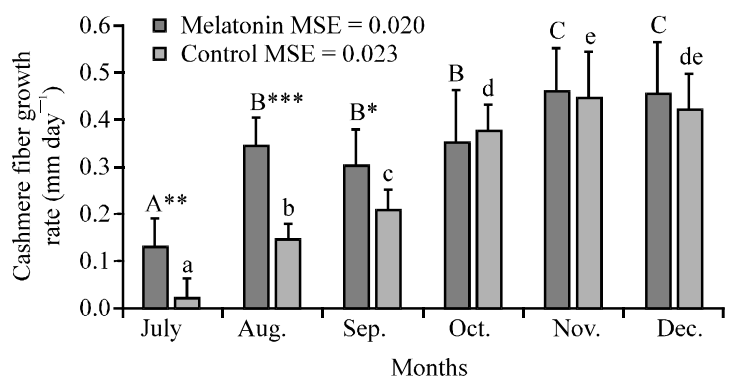

Fig. 2: Both of MT implants groups and control groups mean cashmere fibre growth rate $\left(\mathrm{mm} \mathrm{day}^{-1}\right)$ from July to December. With different capital letters are significant differences $(\mathrm{p}<0.05)$ in the $M$ groups; with different small letter are significant differences $(\mathrm{p}<0.05)$ in the $\mathrm{C}$ groups. The $\mathrm{M}$ groups with ${ }^{*}$ are significant difference $(\mathrm{p}<0.05)$ to the $C$ groups $\left({ }^{* *} \mathrm{p}<0.01 ;{ }^{* * *} \mathrm{p}<0.001\right)$

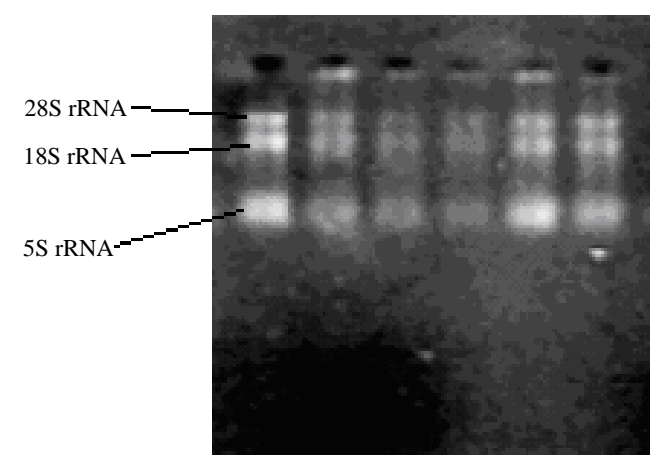

Fig. 3: Result of agarose gel electrophoresis of total RNA from skin of cashmere goats 


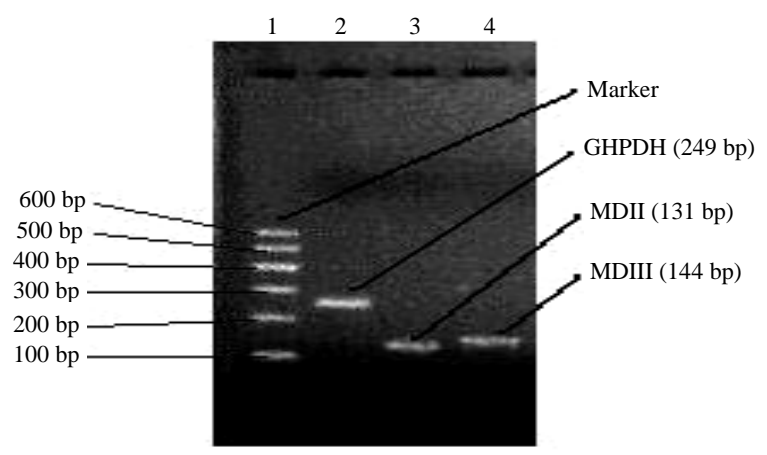

Fig. 4: Identification of real-time PCR products. Sizes of PCR products for GADPH (249 bp), MDII (131 bp) and MDIII (144 bp) were validated using agarose gel electrophoresis

electrophoresis. The results showed that the primers of GADPH, MDII and MDIII were practicable and optimum for real-time quantitative PCR; sizes of PCR products match the theoretically designed length. Dissociation curve plots (melting temperature analysis) showed only one peak for each product. Thus, indicating that specific amplification formation had occurred during the PCR. Linearity and efficiency of PCR amplification were deduced from the given standard curves $\left(\mathrm{R}^{2} \geq 0.995\right)$ by ABI prism 7000 SDS Software (ABI, USA).

The results show that in the $\mathrm{C}$ group cashmere goat skin MDII mRNA from June to November, the expression of a certain regularity from June to September is gradually increased then decreased gradually in August and September the expression was significantly higher than in October, November and June however, after the implant MT MDII mRNA expression increased rapidly, $\mathrm{M}$ and $\mathrm{C}$ groups in July was significantly different $(p<0.01)$ and August are still significant differences $(p<0.05)$ then no significant difference each month. Seen from the results, the early growth of hair in the skin of the MDII mRNA expression is high and then the expression was reduced into normal growth (Fig. 5a).

From June to November, the $\mathrm{M}$ and $\mathrm{C}$ group MDIII mRNA expression showed a downward trend in the cashmere goat skin. The $\mathrm{M}$ group and the $\mathrm{C}$ group did not appear in the monthly significant differences ( $>>0.05$ ). Seen from these results from cashmere to begin to grow into strong growth period the MDIII mRNA expression showed a gradual decrease of regularity in the cashmere goat skin (Fig. 5b).

An important role of MT is to mediate the regulation of seasonal rhythms by photoperiod in mammals (Vanececk, 1998). The results show that in Chinese Inner Mongolia cashmere goats, there were seasonal variations in plasma MT concentration with increasing from the

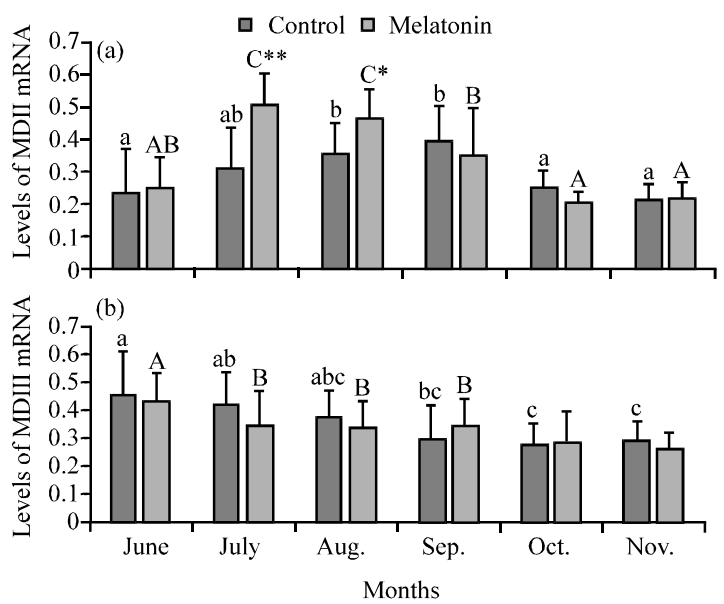

Fig. 5: a) The expression levels of MDII mRNA and b) MDIII mRNA in cashmere goats skin at monthly intervals (The samples of June was sampling before implants of MT) from June to November for both of MT implants groups ( $\mathrm{M}$ groups) and control groups (C groups). With different capital letters are significant differences $(\mathrm{p}<0.05)$ in the $\mathrm{M}$ groups with different small letter are significant differences $(\mathrm{p}<0.05)$ in the $\mathrm{C}$ groups. The $\mathrm{M}$ groups superscribed with $*$ are significant difference $(\mathrm{p}<0.05)$ to the $\mathrm{C}$ groups $\left({ }^{* *} \mathrm{p}<0.01\right)$

June to November for both groups. The data also show that there were significantly enhanced plasma MT concentrations as a consequence of the $\mathrm{MT}$ implants (Fig. 1). These results are similar to previous reports (Nixon et al., 1993; Santiago-Moreno et al., 2005). This is true, the experiment supports the viewpoint that MT has an effect on cashmere fibre growth, especially during the 3 month period after MT treatments, these results shown in Fig. 2. Similarly, the exogenous MT has a positive role on cashmere growth, resulting in increased cashmere production in some breeds (Teh et al., 1991; Jia et al., 1996), the consistent conclusion was obtained in Chinese Inner Mongolia cashmere goats (Wang et al., 2006). Researchers speculated that the MT as a result of the rapid growth of cashmere may be by changing the gene expression of enzyme to cause in the Cashmere goat skin.

Real-time quantitative PCR is a sensitive method that allowed us to use low amounts of starting copy DNA (cDNA) and amplify it to quantitative levels (Bustin, 2000). So, it was used to determine mRNA expression levels in this research. The results show that in the cashmere goat skin MDII mRNA is gradually increased from June to September. Just in this period, the growth of cashmere fiber is also in the growth of start-up period, researchers speculate that this may explain MT increases 
the skin MDII mRNA expression. However, makes us more excited is the early growth of cashmere fiber while MDII mRNA expression was also significantly increased after the MT implants. For example, the group showed that after the implant MT MDII mRNA expression increased rapidly, $\mathrm{M}$ and $\mathrm{C}$ groups in July was significantly different $(\mathrm{p}<0.01)$ and August are still significant differences $(\mathrm{p}<0.05)$ (Fig. 5a). Synchronously, the early rapid growth of cashmere fiber is started after the implanted MT in the same period. For example, the M group cashmere fibre growth rate was markedly greater than that of the $\mathrm{C}$ group in July $(\mathrm{p}<0.01)$, August $(p<0.001)$ and September $(p<0.05)$ (Fig. 2). Therefore, researchers speculate that the rapid growth of cashmere fiber and MDII mRNA was highly expressed may have relevance. In addition, the results show that in Chinese Inner Mongolia cashmere goat skin MDIII mRNA expression of the $\mathrm{M}$ group and the $\mathrm{C}$ group were not appear in the monthly significant differences and from cashmere to begin to grow into normal growth period in the skin MDIII mRNA expression showed a gradual decrease of regularity (Fig. 5b). This shows that the MDIII may not be involved in the physiological process of hair start.

From earlier studies have shown that the growth rate of cashmere and plasma Thyroid hormone (T3, T4) were positively correlated (Rhind and McMillen, 1995; Rhind et al., 2004) found seasonal variation in MD expression in Scottish cashmere goats with a lower level of activity of MDII during periods of long daylength (July and April) and a higher level in December. In later, we have research to prove that the skin $\mathrm{MD}$ activity showed a seasonal variation in Chinese Inner Mongolia cashmere goats with the MDII activity increasing and the MDIII and MDIII/MDII ratio decreasing from June to November (Yue et al., 2007). Until now, researchers found that the rapid growth of cashmere fiber and MDII mRNA was highly expressed have relevance.

Through the above argument therefore, we strongly recommend: from the initiation of cashmere fibre growth to active growth MDII mRNA was highly expressed had a positive effect.

\section{CONCLUSION}

This study shows that along with the start of cashmere fiber growth in Chinese Inner Mongolia cashmere goats MDII mRNA expression levels in the skin gradually increased from June to September and after the implant of MT along with cashmere fiber quick-start growth in the skin while MDII mRNA expression level in the rapid increase in July and August. However, goat skin
MDIII mRNA expression did not appear significantly different from June to November. MDIII mRNA expression in the skin is not sensitive to the implant of MT but the MDIII mRNA expression was gradually decreasing trend from the initiation of cashmere fibre growth to normal growth. These results suggest that the expression levels of MDII mRNA may be involved in the process of cashmere growth, it expression may have a specific signaling pathway in cashmere goats skin, especially in the initiation of cashmere fibre growth period.

\section{ACKNOWLEDGEMENT}

The researchers thank staffs of the stock farm of Inner Mongolia for technical help and cooperation.

\section{REFFRENCES}

Bustin, S.A., 2000. Absolute quantification of mRNA using real-time reverse transcription polymerase chain reaction assays. J. Mol. Endocrinol., 25: 169-193.

Dicks, P., A.J. Russel and G.A. Lincoln, 1994. The role of prolactin in the reactivation of hair follicles in relation to moulting in cashmere goats. J. Endocrinol., 143: 441-448.

Dicks, P., C.J. Morgan, P.J. Morgan, D. Kelly and L.M. Williams, 1996. The localisation and characterisation of insulin-like growth factor-I receptors and the investigation of melatonin receptors on the hair follicles of seasonal and nonseasonal fibre-producing goats. J. Endocrinol, 151: 55-63.

Hynd, P.I., 1994. Follicular determinants of the length and diameter of wool fibres. 2. Comparison of sheep differing in thyroid hormone status. Aust. J. Agric. Res., 45: 1149-1157.

Ibraheem, M., H. Galbraith, J. Scaife and S. Ewen, 1994. Growth of secondary hair follicles on the Cashmere goat in vitro and their response to prolactin and melatonin. J. Anat., 185: 135-142.

Jia, Z.H., T.H. The and M. An, 1996. Effects of exogenous melatonin on the productivity of cashmere goats. Int. Acad. Publ., 2: 890-893.

Kloren, W.R.L. and B.W. Norton, 1995. Melatonin and fleece growth in Australian cashmere goats. Small Ruminant Res., 17: 179-185.

Kloren, W.R.L., B.W. Norton and M.J. Waters, 1993. Fleece growth in Australian cashmere goats. III. The seasonal patterns of cashmere and hair growth and association with growth hormone, prolactin and thyroxine in blood. Aust. J. Agric. Res., 44: 1035-1050. 
Nixon, A.J., V.J. Choy, A.L. Parry and A.J. Pearson, 1993. Fiber growth initiation in hair follicles of goats treated with melatonin. J. Exp. Zool., 267: 47-56.

Rhind, S.M. and S.R. McMillen, 1995. Seasonal changes in systemic hormone profiles and their relationship to patterns of fibre growth and molting in goats of contrasting genotypes. Aust. J. Agric. Res., 46: 1273-1283.

Rhind, S.M., C.E. Kyle and E.I. Duff, 2004. Effects of season and of manipulation of circulating prolactin concentrations on deiodinase activity in cashmere goat skin. Aust. J. Agric. Res., 55: 211-221.

Santiago-Moreno, J., A. Gomez-Brunet, A. GonzalezBulnes, A. Toledano-Diaz, B. Malpaux and A. LopezSebastian, 2005. Differences in reproductive pattern between wild and domestic rams are not associated with inter-specific annual variations in plasma prolactin and melatonin concentrations. Domestic Anim. Endocrinol., 28: 416-429.

Santiago-Moreno, J., A. Lopez-Sebastian, A. del Campo, A. Gonzalez-Bulnes, R. Picazo and A. Gomez-Brunet, 2004. Effect of constant-release melatonin implants and prolonged exposure to a long day photoperiod on prolactin secretion and hair growth in mouflon (Ovis gmelinimusimon). Domestic Anim. Endocrinol., 26: 303-314.
Teh, T.H., Z.H. Jia, K.D. Odgen and G.I. Newton, 1991. The effects of photoperiod and melatonin implant on cashmere production. J. Anim. Sci., 69: 496-496.

Vanececk, J., 1998. Cellular mechanisms of melatonin action. Physiol. Rev., 78: 687-721.

Villar, D., F. Nicol, J.R. Arthur, P. Dicks, A. Cannavan, D.G. Kennedy and S.M. Rhind, 2000. Type II and type III monodeiodinase activites in the skin of untreated and propylthiouracil-treated cashmere goats. Res. Vet. Sci., 68: 119-123.

Wang, L.F., D.X. Lu, H.Z. Sun, X.Y. Zhao and D. San, 2006. Effects of photoperiod and melatonin on nitrogen partitioning and cashmere growth in inner mongolia white cashmere goats. Agric. Sci. Chin., 39: 1004-1010.

Yue, C., L. Du, X. Zhu, X. Kong, W. Zhang and Z. Jia, 2007. Skin deiodinase profiles after melatonin manipulated in Chinese inner mongolia cashmere goats. Asian-Aust. J. Anim. Sci., 10: 1496-1502.

Zhou, H.M., D. Allain, J.Q. Li, W.G. Zhang and X.C. Yu, 2003. Effects of non-genetic factors on production traits of Inner Mongolia cashmere goats in China. Small Rumin. Res., 47: 85-89. 